\title{
Specification of ACMG/AMP guidelines for variant interpretation in Familial Hypercholesterolemia
}

Joana R. Chora ${ }^{1,2}$, Michael A. lacocca ${ }^{3}$, Alain Carriée,5, Sarah E. Leigh ${ }^{6}$, Lukáš Tichý7, C. Lisa Kurtz ${ }^{8}$, Tomas Freiberger ${ }^{9,10}$, Eric J. Sijbrands ${ }^{11}$, Robert A. Hegele ${ }^{3}$, Joshua W. Knowles ${ }^{12,13}$, Mafalda Bourbon ${ }^{1,2}$.

${ }^{1}$ Instituto Nacional de Saúde Doutor Ricardo Jorge, Lisbon, Portugal. ${ }^{2}$ Biosystems and Integrative Sciences Institute, Lisbon, Portugal. ${ }^{3}$ Western University, London ON, Canada. ${ }^{4}$ Hôpitaux Universitaires Pitié-Salpêtrière/Charles-Foix, Paris, France. ${ }^{5}$ Sorbonne Université, Paris, France. ${ }^{6}$ Genomics England, London, United Kingdom. ${ }^{7}$ Center of Molecular Biology and Gene Therapy, University Hospital Brno, Brno, Czech Republic. ${ }^{8}$ University of North Carolina, Chapel Hill NC, USA. ${ }^{9}$ Centre for Cardiovascular Surgery and Transplantation, Brno, Czech Republic. ${ }^{10}$ Masaryk University, Brno, Czech Republic. ${ }^{11}$ Erasmus University Medical Center, Rotterdam, The Netherlands. ${ }^{12}$ Stanford University, Palo Alto CA, USA. ${ }^{13}$ FH Foundation, Pasadena CA, USA.

\section{Background and Aims}

The general ACMG/AMP guidelines for standardized variant interpretation in Mendelian disorders are a great asset in determining variants' pathogenicity, but need to be adapted to each specific gene and disease.

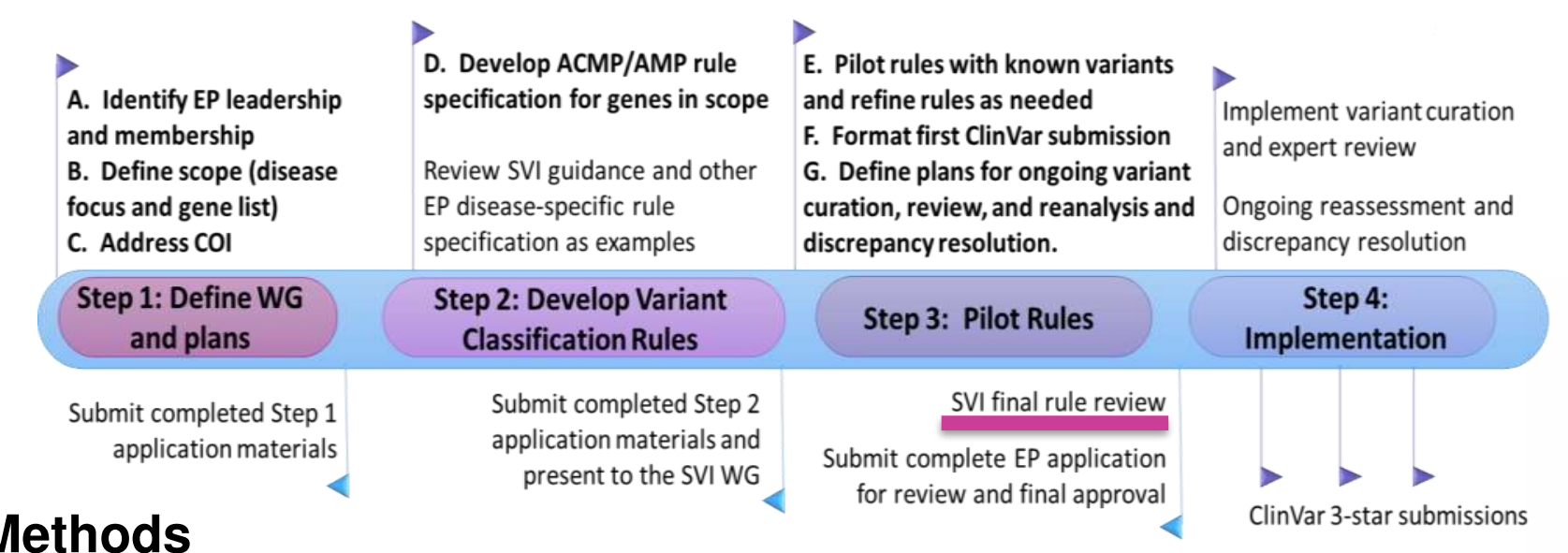

Methods

In 2016, the Clinical Genome Resource consortium (ClinGen) familial hypercholesterolemia variant curation expert panel (FH-VCEP) was created with the goal to specify the guidelines for $\angle D L R, A P O B$ and $P C S K 9$ in $\mathrm{FH}$. The panel of international $\mathrm{FH}$ experts proposed and voted on a draft of specifications, which were tested on known variants. Final adjustments were made based on the pilot study data.

\section{Population data}

For MAF values, use the population with Novel missense variant at the same codon the maximum value in the Genome as a pathogenic (FH-VCEP) variant and Aggregation Database (gnomAD)

- MAF $>0.005(0.5 \%) \rightarrow$ STAND-ALONE (BA1) - MAF $>0.002(0.2 \%) \rightarrow$ STRONG (BS1)

- MAF $<0.0003(0.03 \%) \rightarrow$ MODERATE (PM2) Variant identified in a panel of wellphenotyped normolipidemic controls (minimum of 100 alleles) $\rightarrow$ STRONG (BS2)

\section{Protein specific}

MODERATE (PM1), Mutational hot spot All variants in exon 4, except synonymous MODERATE (PM4), Protein length changes

In-frame indel smaller than whole exon, plus all whole-exon duplications. Variant must meet PM2 criterion.

\section{Cis/trans variants}

When case-level data is available, the identification of a novel variant in a patient: - With homozygous FH phenotype (untreated LDL-C $\geq 13 \mathrm{mmol} / \mathrm{L}$ or $\geq 500$ $\mathrm{mg} / \mathrm{dl}$ ) but only one heterozygous proven pathogenic variant in $L D L R, A P O B$ and PCSK9. Novel variant must meet PM2 criterion $\rightarrow$ MODERATE (PM3)

- With heterozygous $\mathrm{FH}$ phenotype (untreated LDL-C $<13 \mathrm{mmol} / \mathrm{L}$ or $<500$ $\mathrm{mg} / \mathrm{dl}$ ) and one heterozygous proven pathogenic variant in $\angle D L R, A P O B$ and PCSK9 $\rightarrow$ SUPPORTING (BP2)

\section{Not applicable}

Previous report (PP5/BP6); in silico in synonymous (BP7); repetitive regions (BP3) missense in gene with low rate of missense variants (PP2); other mechanisms (BP1/BP5)

With thanks to the EAS for support in the form of a Young Investigator Fellowship. The contents of this poster are presented on behalf of the ClinGen FH Variant Curation Committee.

JR Chora is funded by SFRH/BD/108503/2015. ClinGen is primarily funded by the National Human Genome Research Institute (NHGRI) (grants U41HG006834, U41HG009649, U41HG009650). ClinGen also receives support for content curation from the Eunice Kennedy Shriver National Institute of Child Health and Human Development (NICHD) (grants U24HD093483, U24HD093486, U24HD093487). The content is solely the responsibility of the authors and does not necessarily represent the official views of the National Institutes of Health.

\section{Results}

Here we present the final FH-VCEP proposed guidelines for standardized $\mathrm{FH}$ variant interpretation, although some criteria are still under discussion (!). After debating the pilot study results and the three genes different characteristics, it was decided to focus the full $\mathrm{FH}$-specific guidelines on $L D L R$ alone and set some recommendations on how to interpret $A P O B$ and PCSK9 variants.

\section{Functional data}

\section{STRONG (PS3/BS3)}

Study of LDLR whole cycle in true homozygous patient cells or heterologous cells transfected with the mutant plasmid.

$70 \%$ of wild-type in expre
OR internalization $\rightarrow$ PS3

90\% of wild-type in exp
AND internalization $\rightarrow$ BS3

RNA studies (RNA from patient's cells) with aberrant transcripts quantification. Aberrant transcript is proved by sequencing and is:

$$
\text { 7\% of wild-type transcript } \rightarrow \text { PS3 }
$$

Luciferase studies. Transcription levels: $25 \%$ of wild-type $\rightarrow$ PS3

\section{MODERATE (PS3_Moderate)}

Study of only part of the LDLR cycle in true homozygous patient cells or in heterologous cells transfected with the mutant plasmid.

$<70 \%$ of wild-type in expression, binding OR internalization $\rightarrow$ PS3_Moderate Minigene splicing assay. Aberrant transcript is proved by sequencing and: - no wild-type transcript present $\rightarrow$ PS3_Mod Variants with Level 3 functional studies performed by two or more independent labs, with concordant results (must be different assays)

SUPPORTING (PS3_/BS3_Supporting) Study of LDLR cycle in heterozygous patient cells

$-<85 \%$ of wild-type in expression, binding

OR internalization $\rightarrow$ PS3_Supporting

$>95 \%$ of wild-type in expression, binding

AND internalization $\rightarrow$ BS3_Supporting

RNA studies without transcript quantification

- an aberrant transcript is identified by

sequencing $\rightarrow$ PS3_Supporting

no aberrant transcripts are identified by sequencing $\rightarrow$ BS3 Supporting

High-throughput microscopy; Multiplex Assays of Variant Effect (MAVE); deep mutational scanning assays.

\section{Phenotype}

Variants identified in $\mathrm{FH}$ patients, diagnosis based on any validated clinical criteria, ex. Dutch Lipid Clinic Network $(\geq 6)$, Simon Broome (possible/definite), MEDPED; after alternative causes of high cholesterol are excluded $\rightarrow$ SUPPORTING (PP4)

\section{Conclusions}

After ClinGen approval of these final guidelines, the FH-VCEP will curate all $\mathrm{FH}$ variants in ClinVar at a 3-star level, contributing to standardized $\mathrm{FH}$ diagnosis worldwide and ultimately improve patient management and prognosis.

\section{Loss of function}

\section{VERY STRONG (PVS1)}

(1) deletion of full gene

(2) Null variants predicted to undergo NMD: i) nonsense/ frameshift; ii) GTAG 1,2 splice sites that lead to out of frame consequence; iii) deletion of single or multiple exons that lead to out of frame consequence; iv) intragenic duplications proven to occur in tandem, that lead to out of frame consequence

\section{STRONG (PVS1_Strong)}

1) Remaining null variants: i) nonsense/ frameshift; ii) GT--AG 1,2 splice sites; iii) deletions of single or multiple exons;

(2) intragenic duplications presumed to occur in tandem, that lead to out of frame consequence predicted to undergo NMD.

\section{MODERATE (PVS1_Moderate)}

(1) variant in initiation codon

\section{Prevalence}

Variants in unrelated cases with $\mathrm{FH}$ diagnosis Preferably, case data from ClinVar. Variant must meet PM2 criterion

\section{STRONG (PS4)}

15 or more unrelated cases

MODERATE (PS4_Moderate)

6 to 14 unrelated cases

SUPPORTING (PS4_Supporting)

2 to 5 unrelated cases

\section{Segregation}

Co-segregation of the variant with the phenotype in families. Co-seg $=$ affected relatives (LDL-C > 75th percentile) are carriers and unaffected relatives are not carriers

\section{STRONG (PP1 Strong/BS4)}

Co-seg in $>7$ informative meiosis $\rightarrow$ PP1_Strong

Lack of co-seg in $>2$ informative meiosis (Beware of alternative causes) $\rightarrow$ BS4

MODERATE (PP1_Moderate)

Co-seg in 5-6 informative meiosis

SUPPORTING (PP1)

Co-seg in 3-4 informative meiosis 